\title{
Ecological competition and the incidence of Acinetobacter baumannii bloodstream infections in a teaching hospital in Southeastern Brazil
}

\author{
Letícia Chamma Lastoria $^{[1]}$, Sílvia Maria Caldeira ${ }^{[2]}$, Rayana Gonçalves Moreira ${ }^{[1]}$, \\ Renata Tamie Akazawa ${ }^{[1]}$, Júlia Coutinho Maion ${ }^{[1]}$ \\ and Carlos Magno Castelo Branco Fortaleza ${ }^{[1]}$
}

[1]. Departamento de Doenças Tropicais e Diagnóstico por Imagem, Faculdade de Medicina de Botucatu, Universidade Estadual Paulista, Botucatu, SP. [2]. Departamento de Enfermagem, Faculdade de Medicina de Botucatu, Universidade Estadual Paulista, Botucatu, SP.

\begin{abstract}
Introduction: Recently, pathogen ecology has been recognized as an important epidemiological determinant of healthcareassociated infections (HAIs). Acinetobacter baumannii is one of the most important agents known to cause HAIs. It is widespread in healthcare settings and exhibits seasonal variations in incidence. Little is known about the impact of competition with other hospital pathogens on the incidence of A. baumannii infection. Methods: We conducted an ecological study, enrolling patients who presented with healthcare-associated bloodstream infections (HA-BSIs) from 2005 to 2010 at a 450-bed teaching hospital in Brazil. HA-BSIs were said to be present when bacteria or fungi were recovered from blood cultures collected at least three days after admission. Monthly incidence rates were calculated for all HA-BSIs (overall or caused by specific pathogens or groups of pathogens). Multivariate Poisson regression models were used to identify the impacts of the incidence of several pathogens on the incidence of $A$. baumannii. Results: The overall incidence rate of HA-BSI caused by A. baumannii was 2.5 per 10,000 patient-days. In the multivariate analysis, the incidence of HA-BSI caused by A. baumannii was negatively associated with the incidence rates of HA-BSI due to Staphylococcus aureus (rate ratio $[\mathrm{RR}]=0.88 ; 95 \%$ confidence interval $[\mathrm{CI}]=0.80-0.97)$, Enterobacter spp. $(\mathrm{RR}=0.84 ; 95 \% \mathrm{CI}=0.74-0.94)$ and a pool of less common gram-negative pathogens. Conclusions: Our results suggest that competition between pathogens influences the etiology of HA-BSIs. It would be beneficial to take these findings into account in infection control policies.
\end{abstract}

Keywords: Acinetobacter baumannii. Bloodstream infections. Ecological competition.

\section{INTRODUCTION}

The burden of healthcare-associated infections (HAIs) is especially high in developing countries, most of which are in the tropics ${ }^{1}$. Although work processes are a main cause of HAIs ${ }^{2}$, ecological determinants are increasingly recognized to be relevant epidemiological factors. As an example, recent studies report the seasonality of infections caused by gram-negative bacilli in healthcare settings ${ }^{3,4}$.

Acinetobacter baumannii infections are an interesting example of HAIs, with a high incidence in tropical climates ${ }^{5}$, seasonality ${ }^{6}$ and an increasing incidence of multidrug resistance ${ }^{7}$. Acinetobacter baumannii is one of the most common agents

\footnotetext{
Address to: Dr. Carlos Magno Castelo Branco Fortaleza. Depto ${ }^{\circ}$ de Doenças Tropicais e Diagnóstico por Imagem/FMB/UNESP. Distrito de Rubião Junior s/n, 18618-970 Botucatu, SP, Brasil.

Phone: 5514 3880-1284/3880-1291; Fax: 5514 3815-9898.

e-mail: cmfortaleza@uol.com.br

Received 12 July 2014

Accepted 16 October 2014
}

found in HAIs in Brazilian hospitals and poses a particular threat for patients in high-risk units 8.9 .

Several risk factors for nosocomial acquisition of A. baumannii (overall and multi-drug-resistant isolates) have been reported. These factors include the severity of illness and the use of invasive devices and antimicrobials ${ }^{10-12}$. However, many of these risk factors are similar to those reported for other pathogens ${ }^{13}$. Therefore, competition among microorganisms affecting closed populations subjected to the same ecological pressures may be a possibility in this context.

Our study was designed to assess the effects of such competition. We analyzed, from an ecological perspective, the influence of several pathogens on the monthly incidence rates of healthcare-associated bloodstream infections (HA-BSIs) caused by A. baumannii.

\section{METHODS}

\section{Study setting and period}

The study was conducted in a teaching hospital in an interior part of the State of São Paulo. The hospital has 450 active beds and is a tertiary referral hospital for an area with 500,000 
inhabitants. Intensive care units comprise approximately $10 \%$ of hospital beds. The hospital has a microbiology laboratory and an infection control committee. Our study analyzed data over a time span of 72 months, from 2005 through 2010.

\section{Study design}

The study had an ecological design. We examined the monthly incidence rate data for HA-BSIs (overall or caused by specific pathogens or groups of pathogens). The total number number of months for which data were analyzed was 72 . The outcome of interest was the incidence rate of HA-BSIs caused by A. baumannii.

\section{Data and definitions}

A search was performed using the hospital microbiology laboratory database for the period 2005 to 2010 . To generate monthly data, we counted the number of patients with positive blood cultures. Patients with positive cultures (from any site) in the month previous to admission or within the first three days after admission were excluded, in accordance with the Society for Healthcare Epidemiology of America (SHEA) definitions for healthcare-associated pathogens ${ }^{14}$. We also excluded duplications, defined as the isolation of the same agent from different blood cultures obtained from the same patient within a 30-day period.

To generate a database of monthly incidence rates, we defined HA-BSIs on the basis of a single blood culture that tested positive for one or more typical pathogens. For atypical agents (e.g., coagulase-negative staphylococci [CoNS]), two positive blood cultures collected within $24 \mathrm{~h}$ were required ${ }^{15}$. For the purpose of our study, we did not differentiate primary (or catheter-related) bloodstream infections from those secondary to other primary sites.

Data on the durations of hospital stays (patient-days) were recovered from hospital administrative files. Monthly incidence rates were expressed as cases per 10,000 patient-days.

\section{Statistical analysis}

Analysis was performed using the Statistical Package for the Social Sciences (SPSS) version 19.0 (IBM, Armonk, NY, USA). Briefly, we applied univariate and multivariate models of Poisson regression, selecting the monthly incidence of HA-BSIs caused by A. baumannii as the outcome. Infections caused by other agents or groups were included as predictive factors. A value of $p<0.05$ was considered statistically significant. To present the data graphically, we repeated the analysis using linear regression, with similar results.

\section{Ethical considerations}

This study was approved by the local Committee for Ethics in Human Research.

\section{RESULTS}

In the study period, the overall incidence rate of HA-BSI was 15.8 per 10,000 patient-days. Acinetobacter baumannii ranked third in incidence among the etiologic agents, with an incidence of 2.5 per 10,000 patient-days. In the multivariate analysis (Table 1), the incidence rate of $A$. baumannii was

TABLE 1 - Results of Poisson regression models for the associations between the incidences of several pathogens and the incidence of Acinetobacter baumannii.

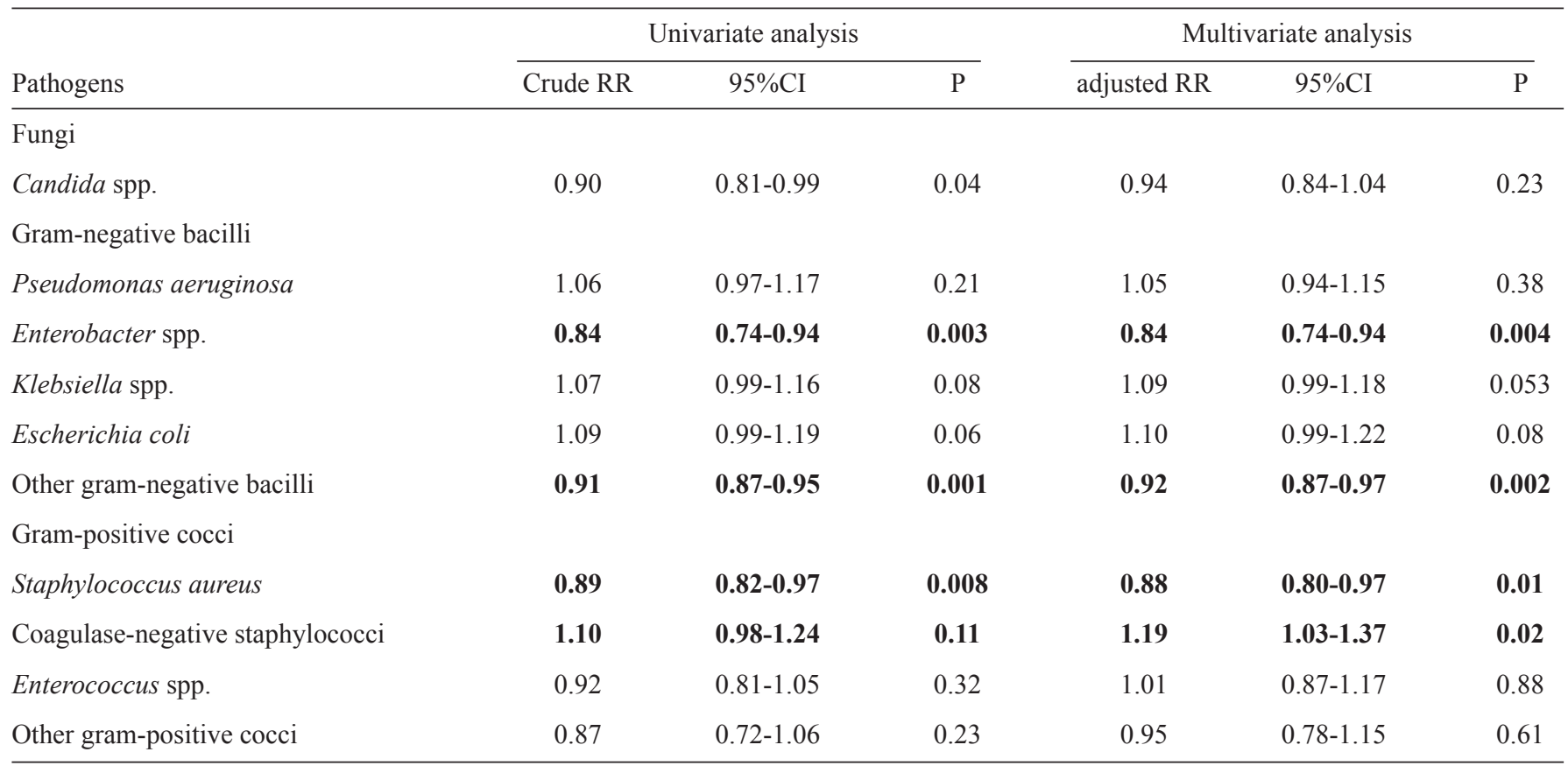

RR: rate ratio, corresponding to the proportional increase (or decrease) in the incidence rate of Acinetobacter baumannii for each unit increase in the incidence of other pathogens. $\mathbf{9 5 \%}$ CI: $95 \%$ confidence intervals. Note: Statistically significant results are presented in bold. 
negatively associated with the incidence rates of Staphylococcus aureus (rate ratio $[\mathrm{RR}]=0.88 ; 95 \%$ confidence interval $[\mathrm{CI}]=0.80-0.97$ ), Enterobacter $\mathrm{spp}$. $(\mathrm{RR}=0.84 ; 95 \% \mathrm{CI}=0.74-0.94)$, and a pool of gram-negative pathogens that individually were of low incidence (e.g., Serratia spp., Citrobacter spp., and several others). We also found a positive association between the incidence of CoNS and A. baumannii.

Although Poisson regression did not presuppose a linear correlation between the incidence rates, linear regression analysis showed a negative impact of Staphylococcus aureus (Figure 1), Enterobacter spp. (Figure 2) and several minor enterobacteria on the incidence $A$. baumannii. To illustrate these relationships, Figure 3 presents the time-series of the monthly incidence rates for $S$. aureus, Enterobacter spp. and A. baumannii.

\section{DIscussion}

Infection control practitioners are faced with several challenges in the control of multi-drug-resistant organisms (MDROs). Guidelines emphasize active surveillance and prompt institution of isolation precautions to contain the spread of MDROs throughout healthcare settings ${ }^{16,17}$. Nevertheless, most current evidence comes from studies focusing on gram-positive pathogens, such as methicillinresistant Staphylococcus aureus (MRSA) and vancomycinresistant enterococci ${ }^{16,18}$. However, a systematic review found insufficient evidence that these recommendations are effective ${ }^{19}$.

The debate becomes more complex when $A$. baumannii ${ }^{20}$ is considered. There are still gaps in our knowledge of the role of antimicrobial use, inanimate reservoirs, asymptomatic colonization and even continuous introduction from environmental sources outside healthcare settings ${ }^{21,22}$. In addition, the risk factors for the acquisition of drug-resistant A. baumannii isolates may vary with the extent of colonization pressure (i.e., the intensity of exposure to other patients harboring this agent) experienced by patients ${ }^{12}$. It is difficult to choose separate, appropriate strategies for fighting $A$. baumannii for each specific setting and period of time.

An ecological approach may help in delineating optimal strategies. We hypothesize that healthcare-associated pathogens compete for reservoirs, both animate and inanimate. Competition between microorganisms has been widely documented in nature ${ }^{23}$ and in the human microbiota ${ }^{24}$. It is plausible that the same phenomenon occurs within hospitals. If so,

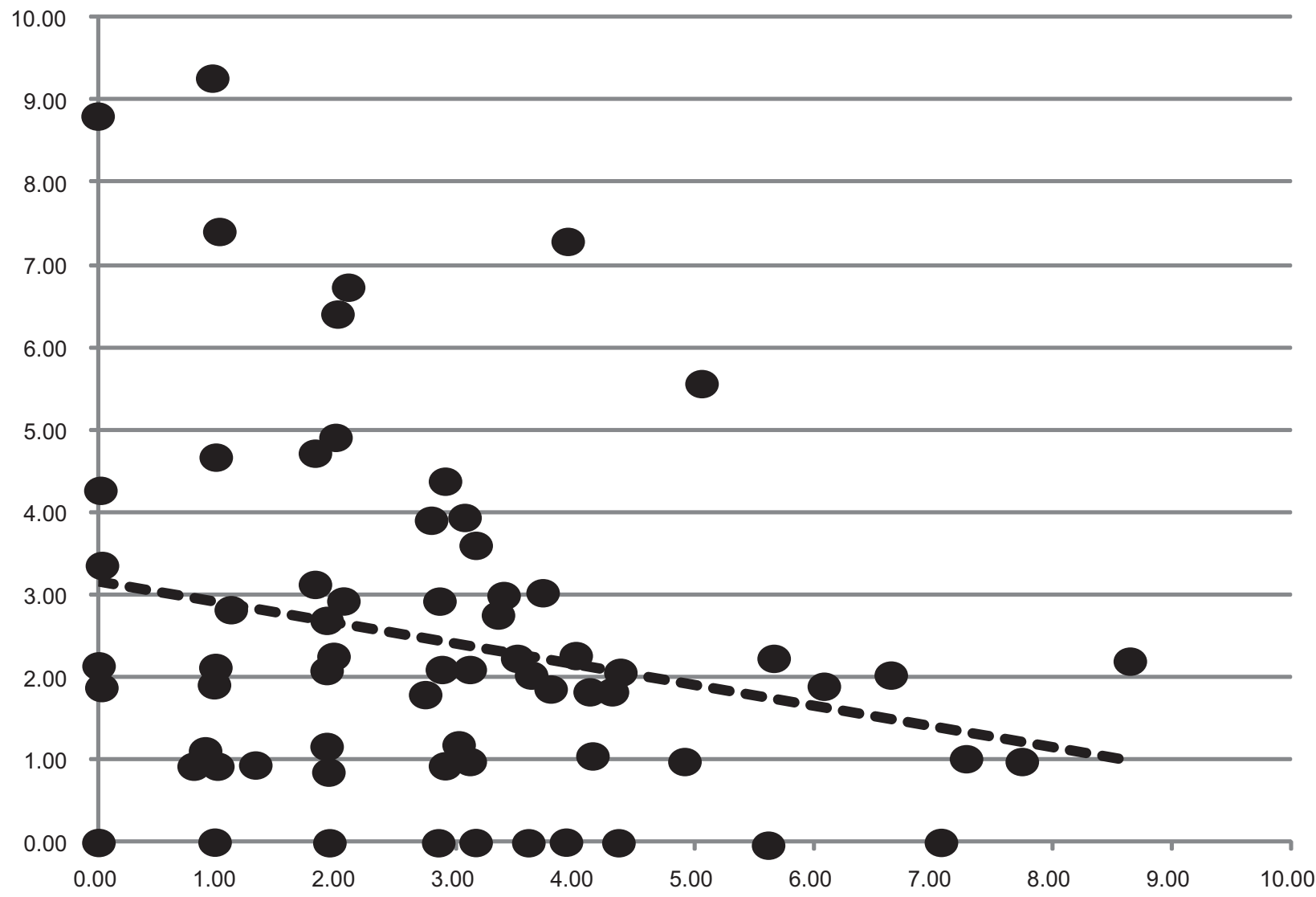

FIGURE 1 - Univariate correlation between the incidences of healthcare-associated bloodstream infections caused by Staphylococcus aureus (x-axis) and Acinetobacter baumannii (y-axis; Pearson's correlation coefficient = -0,23). Note: Incidences are shown as infections per 10,000 patient-days. 


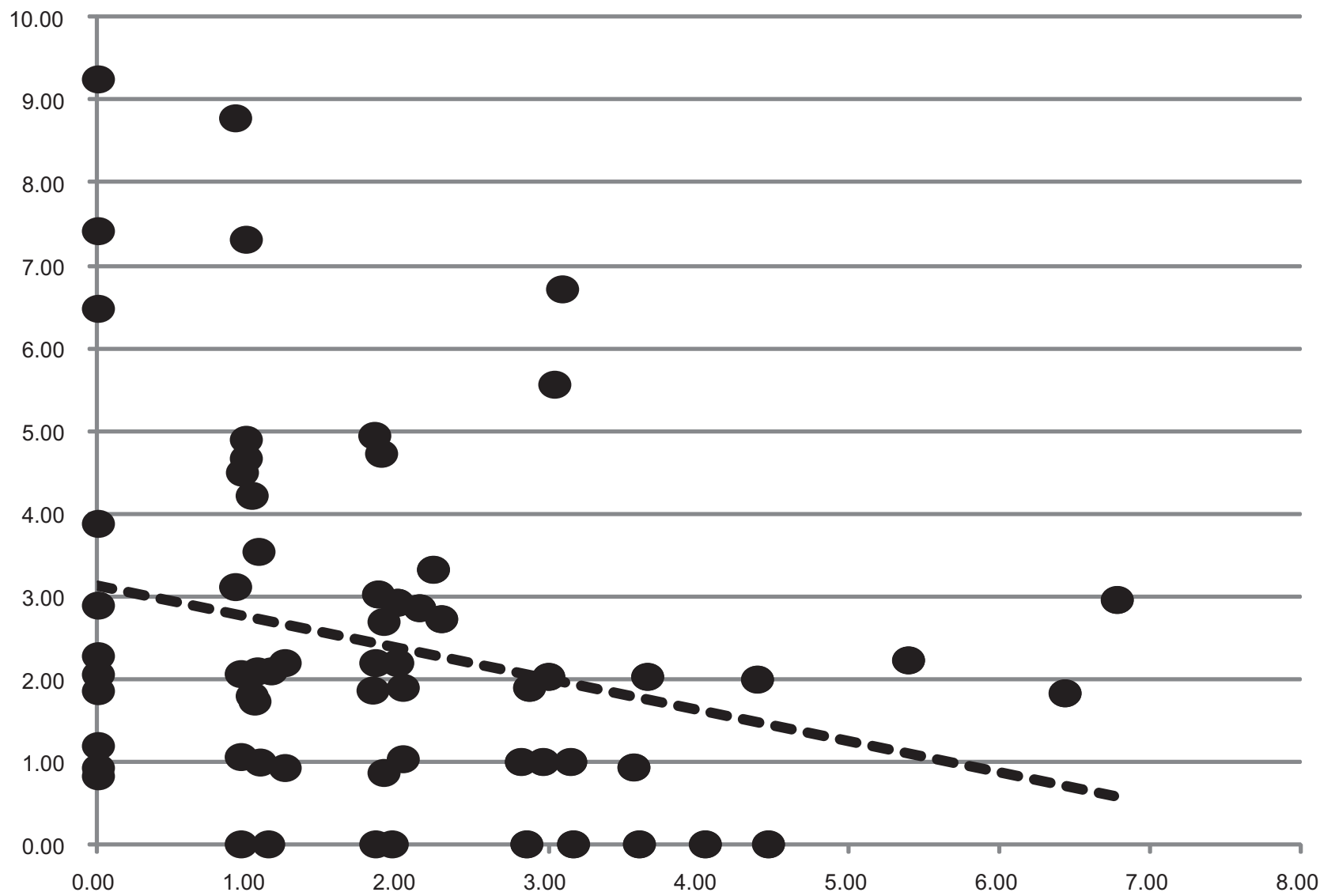

FIGURE 2 - Univariate correlation between the incidences of healthcare-associated bloodstream infections caused by Enterobacter spp. (x-axis) and Acinetobacter baumannii (y-axis; Pearson's correlation coefficient=-0,26). Note: Incidences are shown as infections per 10,000 patient-days.

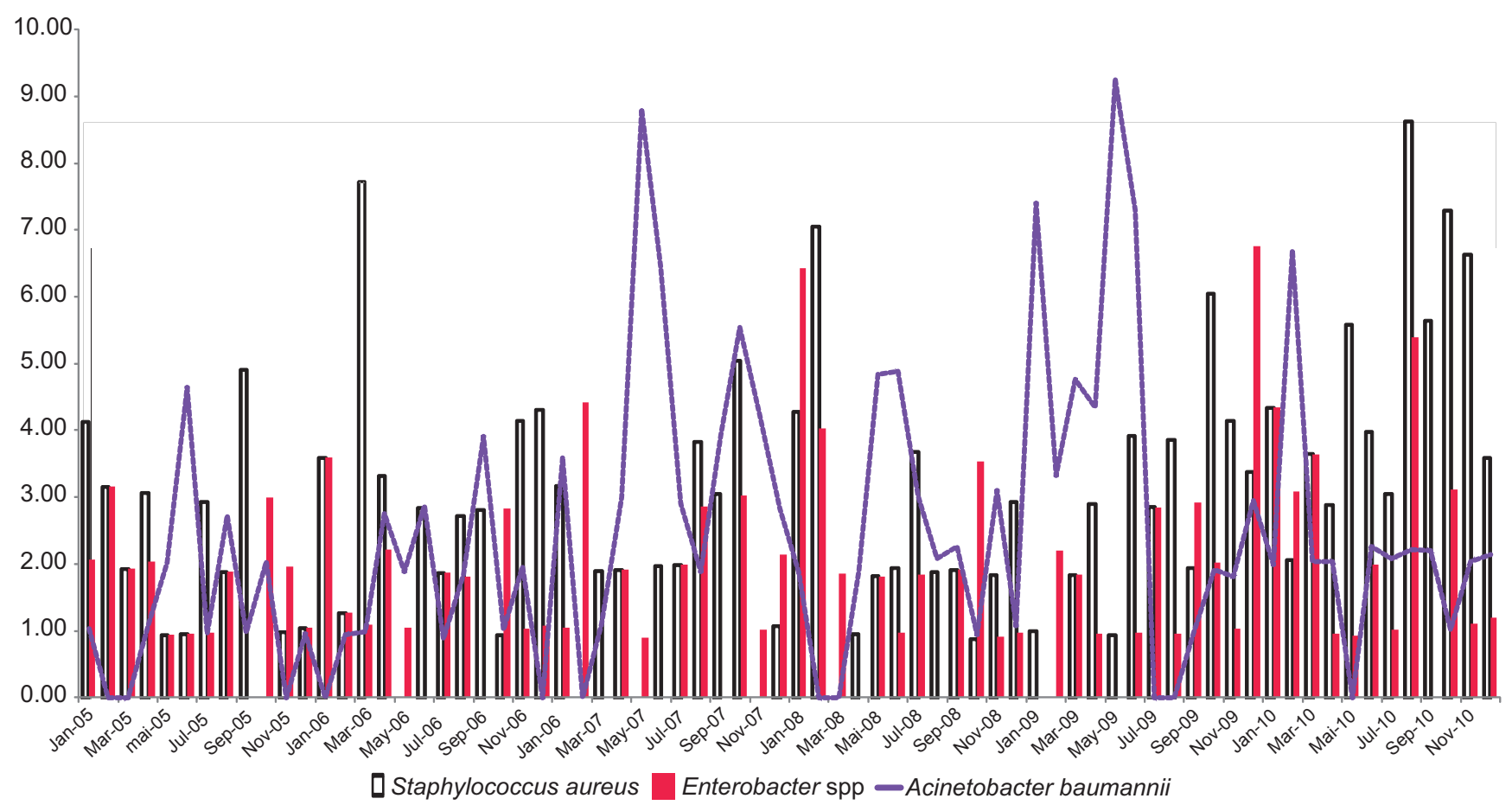

FIGURE 3 - Time series of the incidence (per 10,000 patient-days) of bloodstream infections caused by Staphylococcus aureus, Enterobacter spp. (bars) and Acinetobacter baumannii (line). 
we can estimate trends in the incidence of $A$. baumannii (or any pathogen of interest) in relation to the epidemiology of other microorganisms. An understanding of these relationships may guide the choice of which microorganisms are priority targets for infection control efforts during specific periods. Such an understanding of these relationships may also further our understanding of the behavior of endemic pathogens (e.g., A. baumannii) when challenged with the introduction of emerging agents (e.g., carbapenem-resistant enterobacteria) $)^{25,26}$.

Our study found a negative association between the incidence of $A$. baumannii and the incidences of both $S$. aureus and Enterobacter spp. These findings are noteworthy because they are consistent with our impression, based on experience, that waves of MRSA and multi-drug-resistant A. baumannii infections alternate over time. The finding that competition exists between Acinetobacter baumannii and Enterobacter was unexpected, however, because a previous study in our hospital found that the incidences of both agents (A. baumannii and Enterobacter spp.) are seasonal, with peaks during the warmer months of the year ${ }^{7}$. The negative association between A. baumannii and other, low-incidence gram-negative pathogens was only perceptible when the latter were grouped together, due to a lack of statistical power when they were considered individually. Nevertheless, this finding suggest the possibility that other associations could have been identified if more cases occurred or if the sampling period had been longer. Our study also found a positive association between the incidence rates of CoNS and A. baumannii. While the reasons for this finding are unclear, it suggests that future studies should focus on the competition between staphylococcal species (both coagulasepositive and negative) and A. baumannii.

Our study was limited by the retrospective design and relatively short time span considered. We used a retrospective ecological design, and aggregate data may not be indicative of risks related to individuals (an ecological fallacy). However, ecological analysis is warranted when the implications of interventions are essentially collective, as is the case for many infection control policies ${ }^{27}$. Another limitation of our analysis is that the direction of causality could not be determined in this study. While we found negative correlations between the incidences of pathogens, we cannot be sure whether, for instance, the incidence of $S$. aureus (or Enterobacter spp.) affects that of A. baumannii or whether the opposite occurs. Thus, we must interpret the results in terms of their predictive capability, not in terms of causality. Finally, our study focused only on blood cultures. While this strategy may have lowered the statistical power of our study, we believe that blood cultures are more robust markers of infection than are other microbiological samples.

In conclusion, we found that the incidence of HA-BSIs caused by $A$. baumannii was negatively associated with the incidence of $S$. aureus, Enterobacter spp., and other gramnegative pathogens. Our findings suggest that competition between pathogens occurs in hospital settings and that the effects of this competition should be considered when developing infection control policies.

\section{ACKNOWLEDGMENTS}

RGM and RTA received university student research grants from the Conselho Nacional de Desenvolvimento Cientifico e Tecnológico (CNPq) of the Ministry of Science and Technology in Brazil. JCM received a high school scientific grant (PIBIC Junior) from the same agency.

\section{CONFLICT OF INTEREST}

The authors declare that there is no conflict of interest.

\section{REFERENCES}

1. Allegranzi B, Bagheri Nejad S, Combescure C, Graafmans W, Attar H, Donaldson L, et al. Burden of endemic health-care-associated infection in developing countries: systematic review and meta-analysis. Lancet 2011; 377:228-241

2. Safdar N, Anderson DJ, Braun BI, Carling P, Cohen S, Donskey C, et al. The evolving landscape of healthcare-associated infections: recent advances in prevention and a road map for research. Infect Control Hosp Epidemiol 2014; 35:480-493.

3. Richet H. Seasonality in Gram-negative and healthcare-associated infections. Clin Microbiol Infect 2012; 18:934-940.

4. Schwab F, Gastmeier P, Meyer E. The warmer the weather, the more gram-negative bacteria - impact of temperature on clinical isolates in intensive care units. PLoS One 2014; 9:e91105.

5. Chim H, Tan BH, Song C. Five-year review of infections in a burn intensive care unit: High incidence of Acinetobacter baumannii in a tropical climate. Burns 2007; 33:1008-1014.

6. Fortaleza CM, Caldeira SM, Moreira RG, Akazawa RT, Corrente JE, Souza LR, et al. Tropical healthcare epidemiology: weather determinants of the etiology of bloodstream infections in a Brazilian hospital. Infect Control Hosp Epidemiol 2014; 35:85-88.

7. Zarrilli R, Pournaras S, Giannouli M, Tsakris A. Global evolution of multidrug-resistant Acinetobacter baumannii clonal lineages. Int $\mathrm{J}$ Antimicrob Agents 2013; 41:11-19.

8. Rossi F. The challenges of antimicrobial resistance in Brazil. Clin Infect Dis 2011; 52:1138-1143.

9. Padoveze MC, Assis DB, Freire MP, Madalosso G, Ferreira SA, Valente MG, et al. Surveillance Programme for Healthcare Associated Infections in the State of São Paulo, Brazil. Implementation and the first three years' results. J Hosp Infect 2010; 76:311-315.

10. Jang TN, Lee SH, Huang CH, Lee CL, Chen WY. Risk factors and impact of nosocomial Acinetobacter baumannii bloodstream infections in the adult intensive care unit: a case-control study. J Hosp Infect 2009; 73:143-150.

11. Tan MW, Lye DC, Ng TM, Nikolaou M, Tam VH. Quantifying the effects of risk factors on carbapenem resistant Acinetobacter baumannii: a mathematical modeling analysis. Antimicrob Agents Chemother 2014 Jun 23. pii: AAC.02791-14. PMID: 24957824..

12. Castelo Branco Fortaleza CM, Moreira de Freitas F, da Paz Lauterbach G. Colonization pressure and risk factors for acquisition of imipenemresistant Acinetobacter baumannii in a medical surgical intensive care unit in Brazil. Am J Infect Control 2013; 41:263-265.

13. Safdar N, Maki DG. The commonality of risk factors for nosocomial colonization and infection with antimicrobial-resistant Staphylococcus aureus, enterococcus, gram-negative bacilli, Clostridium difficile, and Candida. Ann Intern Med 2002; 136:834-844. 
14. Cohen AL, Calfee D, Fridkin SK, Huang SS, Jernigan JA, Lautenbach E, et al. Society for Healthcare Epidemiology of America and the Healthcare Infection Control Practices Advisory Committee. Recommendations for metrics for multidrug-resistant organisms in healthcare settings: SHEA/ HICPAC Position paper. Infect Control Hosp Epidemiol 2008; 29:901-913.

15. Horan TC, Andrus M, Dudeck MA. CDC/NHSN surveillance definition of health care-associated infection and criteria for specific types of infections in the acute care setting. Am J Infect Control 2008; 36:309-332.

16. Siegel JD, Rhinehart E, Jackson M, Chiarello L. Healthcare Infection Control Practices Advisory Committee. Management of multidrugresistant organisms in health care settings, 2006. Am J Infect Control 2007; 35 (suppl 2):165-193.

17. Siegel JD, Rhinehart E, Jackson M, Chiarello L. Health Care Infection Control Practices Advisory Committee. 2007 Guideline for Isolation Precautions: Preventing Transmission of Infectious Agents in Health Care Settings. Am J Infect Control 2007; 35 (suppl 2):65-164.

18. Muto CA, Jernigan JA, Ostrowsky BE, Richet HM, Jarvis WR, Boyce JM, et al. SHEA guideline for preventing nosocomial transmission of multidrug-resistant strains of Staphylococcus aureus and enterococcus. Infect Control Hosp Epidemiol 2003; 24:362-386.

19. Aboelela SW, Saiman L, Stone P, Lowy FD, Quiros D, Larson E. Effectiveness of barrier precautions and surveillance cultures to control transmission of multidrug-resistant organisms: a systematic review of the literature. Am J Infect Control 2006; 34:484-494.

20. Rebmann T, Rosenbaum PA. Preventing the transmission of multidrugresistant Acinetobacter baumannii: an executive summary of the
Association for Professionals in infection control and epidemiology's elimination guide. Am J Infect Control 2011; 39:439-441.

21. Zeana C, Larson E, Sahni J, Bayuga SJ, Wu F, Della-Latta P. The epidemiology of multidrug-resistant Acinetobacter baumannii: does the community represent a reservoir? Infect Control Hosp Epidemiol 2003; 24:275-279.

22. Christie C, Mazon D, Hierholzer Jr W, Patterson JE. Molecular heterogeneity of Acinetobacter baumanii isolates during seasonal increase in prevalence. Infect Control Hosp Epidemiol 1995; 16: 590-594.

23. Grover JP1, Hsu SB, Wang FB. Competition between microorganisms for a single limiting resource with cell quota structure and spatial variation. J Math Biol 2012; 64:713-743.

24. Frank DN, Feazel LM, Bessesen MT, Price CS, Janoff EN, Pace NR. The human nasal microbiota and Staphylococcus aureus carriage. PLoS One 2010; 5:e10598.

25. Cabral AB, Melo RC, Maciel MA, Lopes AC. Multidrug resistance genes, including bla (KPC) and bla (CTX)-M-2, among Klebsiella pneumoniae isolated in Recife, Brazil. Rev Soc Bras Med Trop 2012; 45:572-578.

26. Fehlberg LC, Carvalho AM, Campana EH, Gontijo-Filho PP, Gales AC. Emergence of Klebsiella pneumoniae-producing KPC-2 carbapenemase in Paraíba, Northeastern Brazil. Braz J Infect Dis 2012; 16:577-580.

27. Szklo M, Nieto FJ. Epidemiology - beyond the basics. $3^{\text {rd }}$ edition. Burlington, MA: Jones and Bartlett; 2014. 